\title{
CORRESPONDENCE
}

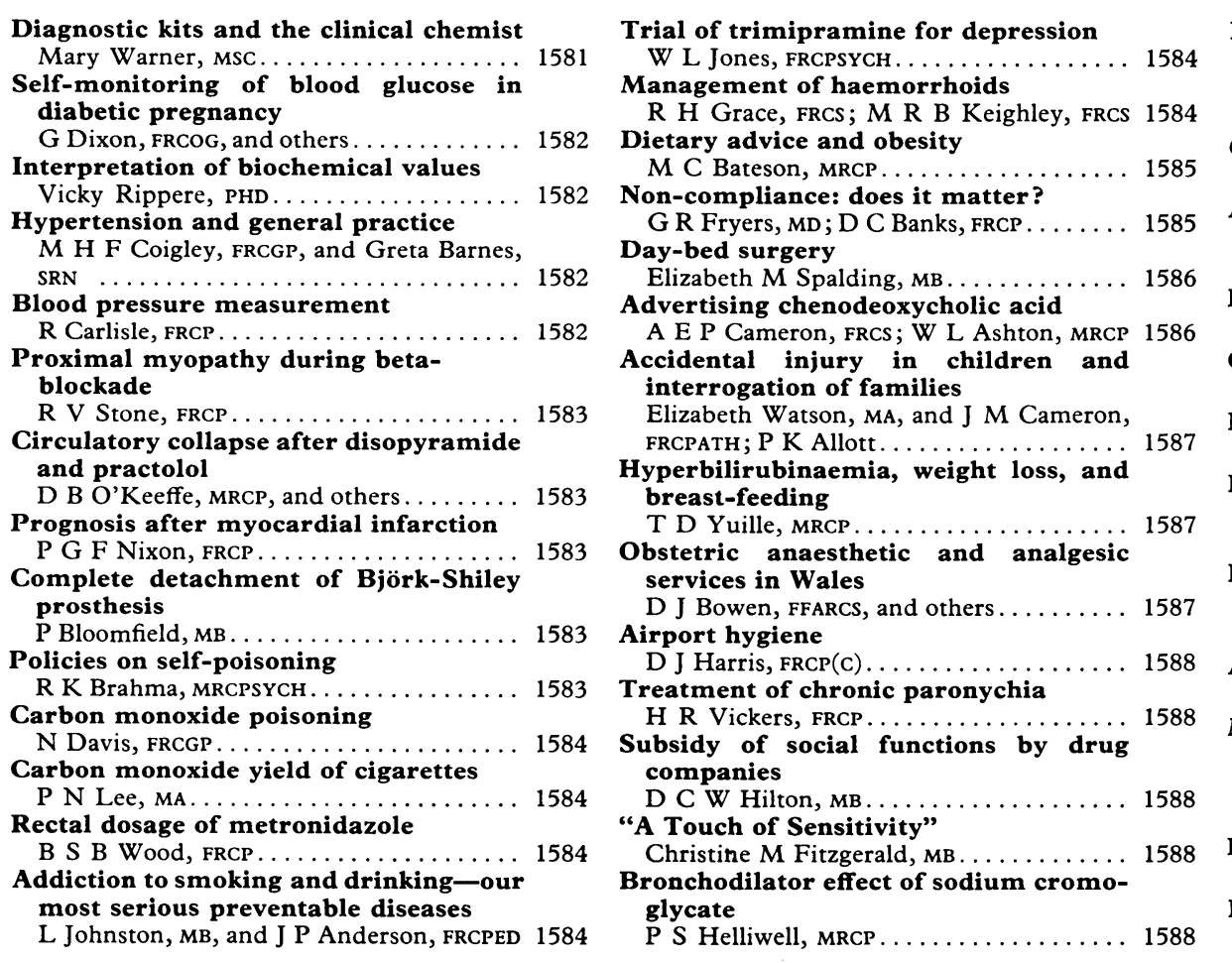

Rubella vaccination and unnecessary

abortions

J W Peters, MRCoG, and Elizabeth G

Dowsett, MB................ 1588

Coal-mining and mortality

A L Cochrane, FRCP............ 1589

Audiological consultantship-a sine-

cure?

A G Gordon. . . . . . . . . . . . . 1589

Inhalation of formaldehyde vapour

A R Lyne. . . . . . . . . . . . . . . . . . . 1589

Guar crispbread in the diabetic diet

N Mantel. . . . . . . . . . . . . . . . . . . . . 1589

If I was forced to cut

D L Freedman, MD . . . . . . . . . . 1589

Hypercalcaemia after tamoxifen for breast cancer

G J G Rees, MRCP. . . . . . . . . . 159

Resuscitation of the newborn

A R J Bosley, MRCP; J C S Ainley-Walker,

FFARCS ................. 1590

Accident and emergency services

W J Morgan, FRCSED . . . . . . . . . . . 1590

Multidisciplinary teams

R T Ritchie, FRCPED; $T$ L Pilkington,

FRCPSYCH; Denise Platt, AIMSW, and

Susan Hill, AIMsw............. 1590

Health visitors and confidentiality

Margaret Rushforth............. 1591

Penis captivus

R Roberts, FRCP(C) . . . . . . . . . . . 1591

We may return unduly long letters to the author for shortening so that we can offer readers as wide a selection as possible. We receive so many letters each week that we have to omit some of them. Letters must be signed personally by all their authors. We cannot acknowledge their receipt unless a stamped addressed envelope or an international reply coupon is enclosed.

\section{Diagnostic kits and the clinical chemist}

SIR,-I was interested in the article on the self-monitoring of blood glucose levels in diabetic pregnancy (24 November, p 1333) and obviously this illustrates a specific advance in patient care. However, this type of test is the tip of an enormous iceberg resting with the manufacturing companies and, as a clinical chemist, I am concerned by certain aspects of tests performed outside laboratories that must be closely monitored.

Clinical chemistry itself has been the main target for diagnostic products and kits for many years and while many of these have been a significant influence on our work pattern and reliability many kits can be shown to be inaccurate and unreliable. As these are entering a laboratory environment in general they are nipped in the bud and quietly expire. Apart from this natural death the DHSS has a clinical chemistry section that can issue Hazard Notes or otherwise prevent the sale of unsuitable products. The reason that poor kits are released in the first place is that manufacturers just do not have at their disposal realistic testing materials or situations. It has taken many years for clinical chemists to present an organised front to these manufacturers, but now that this market is so controlled and particular an easier outlet is being sought. By defining a product as suitable for ward use there is a direct entry into the hospital via pharmacy departments for kits and reagent strips that may or may not be accurate or reliable enough for the use to which they will be put. Manufacturing companies exist to make a profit not to provide the best service for the patient, and before clinicians adopt tests of this nature they need to take informed decisions. The clinicians need to establish whether there is an acceptable alternative and a trip to the local laboratory to discuss an alteration in work pattern may be a start. Laboratories are now emerging from a deluge of work, in the past channelled straight on to inflexible equipment, to ask such questions as whether we are actually providing the service clinicians require. When it has been decided that the laboratory cannot provide a service it is valid to consider the manufacturers' alternatives. The DHSS enters here as it runs evaluations of many products in a realistic fashion. It has, in fact, just completed an evaluation of glucose meters showing that not all are satisfactory. Once a basic validity has been established a local test needs to be performed using the operators who will, in fact, be providing the test results. In this way any rogue tests should be eliminated.

To return more specifically to glucose strips and meters, many clinical chemists would agree that a highly motivated, specifically trained diabetic patient on an adequate meter will be all right. To take this meter into an intensive care unit is, however, a different situation entirely. Here you are faced with multiple operators, probably a poorly maintained machine, and a rapidly changing, metabolically complex patient. Often these patients are incapable of communication and no type of informed consent can be obtained. Within the South-western Region there have been two deaths in cases where incorrect clinical decisions have been based on strip results. There is no comeback to the manufacturers since the glucose stick and meter can be shown to be reliable if correctly stored, used, and operated. The sticks and meters often have relatively poorer performances at the clinically important upper and lower ends of the scale, once again limiting usefulness in the extreme situations liable to be found in an intensive care unit. The strips can vary from manufacturer to manufacturer; the Ames Kinetic type needs a stricter adherence to timing than the Boehringer endpoint type, though in the latter sensitivity is lost at high levels.

And so, in summary, please use the expertise of the British clinical chemist; we have a 
worldwide reputation for our thoroughness and meticulous attention to detail. We have the same aim as clinicians, the benefit of the patient; and united we can extract the best from the manufacturing companies, eliminating dangerous and unsuitable products on the way. In rase I am accused of merely fretting over my livelihood, I must point out that removing glucose completely from my laboratory's repertoire cuts our number of tests from about 120000 to 105000 -and we have one of the smallest laboratories. To remove everything we do means a lot of work for someone somewhere, and is it really what the clinicians want?

M WARNER

Department of Clinical Pathology,

Musgrove Park Hospital,

Taunton,

Somerset TA1 5DA

\section{Self-monitoring of blood glucose in diabetic pregnancy}

SIR,-We have read with great interest the article by $\mathrm{Dr}$ I Peacock and his colleagues (24 November, p 1333) and agree with them that diabetic pregnancy can now best be managed on an outpatient basis. Over the past 12 years we have adopted a very similar regimen for the 153 pregnancies with which we have dealt. The perinatal mortality when corrected for lethal congenital malformations has been $3 \%$.

We have, however, on occasions been worried that the occasional patient was observing her strict dietary and insulin regimen only during the days when she was doing her own outpatient blood glucose monitoring; and more recently we have taken blood for glycosylated haemoglobin estimations each time the patient visits the hospital. We have found this estimation to be of great value in detecting those mothers whose blood glucose control is less than optimal. The mean glycosylated haemoglobin during the final trimester correlates well $(r=0.69, P<0.01)$ with the cord blood C-peptide:glucose ratio, which has been shown ${ }^{1}$ to indicate the degree of hyperresponsiveness of the fetal beta-cells induced by maternal hyperglycaemia. We have also found that the maternal glycosylated haemoglobin at the time of delivery correlates well with the mean blood glucose over the final trimester $(r=0.74, P<0.05)$. We have further found that the glycosylated haemoglobin levels (estimated by the method of Fluckiger $^{2}$ ) below $9 \%$ suggest that a good fetal outcome is likely and that maternal blood glucose control is good.

While supporting the principles outlined by Dr Peacock we do recommend the combination of outpatient blood glucose monitoring and regular measurement of maternal glycosylated haemoglobin levels as the best method of monitoring diabetic pregnancy.

GEOFFrey DixoN

B J BURKE

P E SAVAGE

Department of Obstetrics and Gynaecology,
Bristol Maternity Hospital,
Bristol BS2 8EG

1 Burke, B J, et al, Lancet, 1979, 1, 1372. Fluckiger, R, Bergen, W, and Winterhalter, $\mathrm{K} \mathrm{H}$,

\section{Interpretation of biochemical values}

SIR,-I am writing in the hope that one of your readers may be able to clarify a mysterious and widespread medical practice-namely, drawing the conclusion that because an individual's value on some biochemical investigation, such as thyroxine estimation, is within the population normal range for the particular laboratory the function in question is de facto normal for that individual.

Elsewhere in biometrics, in particular in psychometry, practitioners distinguish between nomothetic (for example, the case in which the individual's value is compared with population norms) and idiographic (for example, the case in which the individual's present value is compared with his own personal norm or baseline) assessment. Thus it is not uncommon to diagnose pathological underfunctioning in the presence of values which may be above average for the population but which are known, or suspected on reasonable grounds, to fall below the norm for that particular individual.

Although writers such as Eastham ${ }^{1}$ point to the lack of any necessary correlation between normal biochemical value and the patient's actual clinical state, I have yet to meet a doctor who appears to act on acceptance of the principle of idiographic assessment, even when baseline data obtained when the patient was asymptomatic are available. Even when the normal range for a particular substance is extremely wide, many complaints from patients whose values fall within that range often seem to be dismissed as unfounded. Can someone enlighten me why this is so ?

VICKY RIPPERE

Department of Psychology,

Institute of Psychiatry,

London SE5 8AF

1 Eastham, R D, Biochemical Values in Clinical Medicine. Bristol, John Wright, 1978.

\section{Hypertension and general practice}

SIR,-Dr H G Nicol's reaction (24 November, p 1368) to Dr D G Beever's little outburst (3 November, $p$ 1137) prompted us to re-read the letter's words and to offer some history, some figures, and the following comments.

One should be very sure of one's facts before making sweeping generalisations. The truth is that all physicians were disgracefully unaware of the importance of detecting and treating hypertension for very many years. The life insurance companies were far ahead of us and long ago showed ${ }^{1}$ that the early middle aged with diastolic pressures of $99 \mathrm{~mm} \mathrm{Hg}$ and over had about a 15 times greater chance of having a stroke by their 65 th year than the normotensive. By 1914 few could obtain a life insurance policy without having their blood pressure recorded.

One of us (MC) has a letter in the files of 15 years ago from a consultant physician advising that no treatment was necessary for a patient with a blood pressure of $220 / 115 \mathrm{~mm} \mathrm{Hg}$ because a well-known professor of cardiology (named) advised no therapy until the diastolic pressure was $120 \mathrm{~mm} \mathrm{Hg}$ or above. This was the teaching which most general practitioners (and consultants) then received. It is only within the last 10 years that the mass of opinion has changed. The interest of one of us (MC) was originally aroused when he realised that so many hypertensives were presenting initially with a stroke. Could early detection prevent this? We have now known for some time that it can. ${ }^{2} 3$

What are the present facts? Some light may be thrown on this question by the analysis of the screening figures obtained by one of us (GB), who is closely concerned with the present Medical Research Council treatment trial for mild-to-moderate hypertension. In 72 general practices 194801 people aged 35-64 years were screened (the response rate being $74 \%$ ). The total found to be mildly hypertensive (systolic pressure $<200 \mathrm{~mm} \mathrm{Hg}$, diastolic $\geqslant 90 \mathrm{~mm} \mathrm{Hg}<110 \mathrm{~mm} \mathrm{Hg}$ ) was 14611 or $7.5 \%$ after four readings, while those with severe hypertension (systolic pressure $\geqslant 200 \mathrm{~mm} \mathrm{Hg}$ or diastolic $\geqslant 110 \mathrm{~mm} \mathrm{Hg}$, or both) were only $0.95 \%$. Moreover, of the total screened (194801) $6.4 \%$ were found to be already on treatment and of these half had diastolic pressures of under $90 \mathrm{~mm} \mathrm{Hg}$.

Surely this does not show general practitioners to be disgracefully underdiagnosing or undertreating hypertension, especially with regard to detection, as a great number of these people had not seen their doctor for some considerable time. Let us therefore stop throwing brickbats and all work towards improving the detection of hypertension (and occult disease) in our general or hospital practices. This, of course, would be aided by any uninvolved general practices inquiring about the MRC trial (Epidemiology and Medical Care Unit, Northwick Park Hospital, Harrow, Middx HA1 3UJ) and maybe taking part if they are suitable.

A little more age and experience will change, we hope, Dr Beever's views.

Michael Coigley Greta Barnes

Stratford-upon-Avon,

CV37 6LR

Metropolitan Life Insurance Survey. New York, 1961. Leishman, A W D, Lancet, , 1963, 1, 1284. E $\mathrm{O}$ Quarterly fournal of Medicine, 1970, 39, 411.

\section{Blood pressure measurement}

SIR,- In the course of seven articles Dr Eoin O'Brien and Professor Kevin O'Malley consider multifarious sources of error in estimating-what? At times it is the instantaneous intra-arterial pressure (systolic and diastolic); at other times a casual reading is maligned and a mean or "representative" blood pressure, from continuous or from home recording, is preferred. Surely the errors resulting from variable definition are greater than those (fortunately often random or mutually opposed) which are addressed in the articles.

If we exclude continuous recordings of home recordings as impractical for the majority of diagnoses and follow-ups, should blood pressure be defined from samples of one, two, or more sets of readings? Their answers vary, though we are consistently advised to take three readings (one systolic and two diastolic) in each set. There are indeed good reasons for three-a single quirky value can be identified; and in this case three values are readily obtainable, with less bias too than if they were supposedly identical.

We also need to know, however, at what 